\title{
L'OCDE évalue les compétences des adultes
}

Anne-Marie Bardi

\section{OpenEdition}

\section{Journals}

Édition électronique

URL : http://journals.openedition.org/ries/3659

DOI : $10.4000 /$ ries.3659

ISSN : 2261-4265

\section{Éditeur}

Centre international d'études pédagogiques

\section{Édition imprimée}

Date de publication : 15 avril 2014

Pagination : 16-19

ISBN : 978-2-85420-603-6

ISSN : 1254-4590

\section{Référence électronique}

Anne-Marie Bardi, «L'OCDE évalue les compétences des adultes », Revue internationale d'éducation de Sèvres [En ligne], 65 | avril 2014, mis en ligne le 15 avril 2017, consulté le 07 janvier 2020. URL : http:// journals.openedition.org/ries/3659; DOI : 10.4000/ries.3659

Ce document a été généré automatiquement le 7 janvier 2020

(c) Tous droits réservés 


\title{
L’OCDE évalue les compétences des adultes
}

\author{
Anne-Marie Bardi
}

Une dizaine d'années après les premiers résultats du Programme international de suivi des acquis des élèves (PISA), l'Organisation de coopération et de développement économiques (OCDE) livre une étude sur les compétences des adultes de 16 à 65 ans. Ce nouveau Programme pour l'évaluation internationale des compétences des adultes (PIIAC) est conçu en cycles, dont le premier s'achève en 2013. Il vise à informer sur la maîtrise des compétences jugées indispensables pour évoluer dans un monde hyper connecté et participer pleinement à l'économie mondialisée du XXI siècle. Des conseils sur les mesures politiques à adopter accompagnent les résultats. Ils reposent sur quelques postulats : le développement économique d'un pays est directement fonction des compétences de ses citoyens; la répartition des compétences au sein de la population a un impact sur les résultats économiques et les comportements sociaux; des décisions politiques éclairées permettent d'améliorer les compétences demandées par un marché du travail en pleine mutation.

2 Les compétences évaluées, appelées «compétences clés en traitement de l'information ", s'organisent en trois domaines : littératie, numératie et résolution de problèmes dans des environnements fortement informatisés. Pour chaque individu évalué, on recueille aussi des données sociodémographiques, des informations sur l'utilisation de ses compétences dans le cadre professionnel, sur l'emploi, le revenu, la santé et l'engagement politique ou social.

\section{Les compétences évaluées}

3 La littératie est la capacité de comprendre, d'évaluer et de s'engager dans des textes écrits. Il s'agit d'identifier une information, de mettre en relation, d'interpréter, d'évaluer des informations. Sont exclus l'expression orale et la production écrite. 
La numératie est la capacité de localiser, d'utiliser, d'interpréter, de communiquer des informations et des concepts mathématiques tels quantité et nombre, dimension et forme, variation, relation, hasard, diagramme, carte, graphique, etc. La résolution de problèmes dans un environnement à forte composante technologique est la capacité d'utiliser les technologies numériques, les outils de communication et les réseaux pour acquérir et évaluer de l'information, communiquer avec autrui et accomplir des tâches usuelles.

\section{Les modalités de l'enquête}

Environ 166000 adultes âgés de 16 à 65 ans ont été interrogés dans 24 pays et entités sous-nationales. La langue de l'évaluation correspondait à la langue officielle du pays de passation. L'évaluation s'est déroulée au domicile du répondant et a été réalisée sur ordinateur portable ou sur papier (carnet de test) selon ses compétences informatiques. Les répondants présentant de très faibles compétences en littératie ont été orientés uniquement vers des tests en "composantes de lecture" (déchiffrage, vocabulaire, etc.). Quatre pays (dont la France) n'ont pas procédé à l'évaluation de la résolution de problème dans des environnements à forte composante technologique.

\section{Les résultats bruts}

7 Six niveaux de compétence ont été définis en littératie. Au niveau le plus faible (inférieur à 1), on demande de trouver une information explicite dans un texte court portant sur un domaine familier. Globalement 3,3\% des adultes se situent à ce niveau (5,3 \% en France). Au niveau 1, il faut lire plusieurs paragraphes et trouver des données réparties dans le document $12,2 \%$ des adultes se situent à ce niveau (16,2 \% en France). Au niveau 5, le plus élevé, on doit pouvoir rechercher plusieurs informations dans des textes denses, évaluer leur fiabilité, les confronter, les synthétiser voire évaluer des arguments et reconnaître des marqueurs rhétoriques subtils. Ce niveau est atteint par $0,7 \%$ de la population en moyenne ( $0,3 \%$ en France).

Cette répartition en six niveaux se retrouve en numératie, avec $5 \%$ de la population globale en dessous du niveau 1 ( $9 \%$ en France), $14 \%$ au niveau 1 (18,9 \% en France) et $1,1 \%$ au niveau 5 ( $0,5 \%$ en France).

Pour la résolution de problèmes dans des environnements à forte composante technologique, certaines personnes ne passent pas le test de base en informatique (15\% ne peuvent utiliser la souris ou le clavier, auxquels s'ajoutent $10 \%$ qui ne souhaitent pas travailler sur ordinateur) ; les autres se répartissent en quatre niveaux : inférieur à $1,1,2$ et 3 . Le test propose des tâches numériques « courantes " liées à la messagerie, à l'achat en ligne, aux forums, etc.

10 Si l'on classe les pays selon le pourcentage de la population « compétente ", c'est-à-dire située aux niveaux 3,4 ou 5 , en littératie, le Japon arrive en tête $(71 \%$ de sa population), suivi par la Finlande, les Pays-Bas, la Suède, l'Australie, la Norvège (55\%). En queue de classement, on trouve la France (42\%), l'Espagne (33\%) et l'Italie (29\%). Si l'on s'intéresse à la numératie, on trouve également en tête le Japon ( $62 \%$ de sa population aux niveaux 3 et plus), suivi de la Finlande, de la Suède, des Pays-Bas et de la Norvège, tandis qu'en queue de liste se situent la France (37 \%), l'Irlande, les États-Unis, 
l'Italie et l'Espagne (27\%). Pour la résolution de problèmes dans un environnement numérique arrivent en tête la Suède, la Finlande, les Pays-Bas, la Norvège (plus de $40 \%$ de la population aux niveaux 2 et 3), tandis que la Pologne n'atteint pas $20 \%$.

\section{L'impact des critères sociodémographique}

11 Les moyennes ne rendent pas compte des disparités internes; les informations complémentaires permettent d'affiner les analyses et de présenter des résultats croisés ou ajustés.

\section{L'effet de l'âge}

12 L'écart de performances entre les 16-24 ans et les 55-65 ans est toujours en faveur des cadets. Il tient aux évolutions économiques, à la généralisation de la scolarisation, aux possibilités de formation continue, à la mobilisation des compétences dans le monde du travail et, pour ce qui est de la résolution de problèmes dans un environnement technologique, au fait d'avoir vécu depuis le plus jeune âge dans un monde où les technologies de l'information sont très présentes. En littératie, la Corée connait l'écart le plus élevé, avec des seniors parmi les moins performants de leur classe d'âge et des jeunes parmi les plus performants. Cet écart est également très important en Finlande et en France : dira-t-on que le niveau monte ? À l'opposé, l'Angleterre/Irlande du Nord, la République slovaque ou les États-Unis connaissent des écarts de performance très minimes entre les jeunes et les plus âgés, ce qui peut tenir à un niveau élevé de compétence chez ces derniers.

\section{Hommes-femmes}

En littératie, les différences entre les sexes, généralement en faveur des hommes, disparaissent dans les populations plus jeunes et lorsqu'elles sont statistiquement significatives c'est en faveur des femmes (Estonie, Pologne). En numératie comme en résolution de problèmes, les scores des hommes sont supérieurs à ceux des femmes mais, pour les populations les plus jeunes, l'écart s'estompe dans la moitié des pays. Ce constat est à rapprocher de la différence d'utilisation des TIC dans les pratiques professionnelles, plus fréquente pour les hommes que pour les femmes dans quinze pays.

\section{Le niveau de formation initiale}

14 L'enquête distingue trois niveaux de formation initiale: inférieur au "secondaire supérieur (lycée) », lycée et enseignement supérieur. La différence de score entre les deux extrêmes est la plus forte aux États-Unis (67 points en littératie, 83 points en numératie) suivie par la France (63 points et 79), tandis que la différence la plus faible s'observe en Estonie (33 points et 42 ). Au Japon, les compétences des personnes à faible formation initiale dépassent, en littératie, celles des Français ou des Américains de formation initiale moyenne. 


\section{Le milieu socio-économique}

L'étude utilise le niveau de formation des parents comme indicateur du milieu socioéconomique et constate, sans surprise, une corrélation positive entre le niveau de performance et le milieu socioéconomique. Toutefois cette corrélation varie beaucoup d'un pays à l'autre et, dans le même pays, d'une tranche d'âge à l'autre. Si l'on compare le score moyen en littératie des personnes ayant au moins un parent diplômé de l'enseignement supérieur et celui des personnes dont aucun parent n'est diplômé de l'enseignement secondaire supérieur, on observe les plus grandes différences en France, en Pologne, en Allemagne et aux États-Unis, les plus faibles en Estonie, en Australie et au Japon. On retrouve là des tendances déjà constatées par PISA, avec un plus fort marquage social en France notamment. Pour la résolution de problèmes en milieu technologique, $12 \%$ des personnes issues de milieux favorisés atteignent le niveau 3 (plus de $15 \%$ en République tchèque, en Finlande et en Suède) tandis qu'ils sont moins de $2 \%$ parmi les personnes issues de milieux défavorisés.

\section{Les effets de l'immigration}

Il n'est pas surprenant que les immigrés de langue étrangère soient moins compétents que les autochtones. Les différences observées entre immigrés récents et immigrés établis traduisent l'efficacité des politiques d'intégration, voire un changement de politique d'immigration (en Autriche, les derniers arrivés sont plus performants que les anciens).

\section{Compétences, vie professionnelle et vie sociale}

Sur la base d'auto-déclarations, PIIAC a recensé les compétences en traitement de l'information et les compétences dites génériques utilisées dans le cadre professionnel (hiérarchiser ses tâches, organiser, apprendre, coopérer, etc.), ce qui permet d'identifier des différences notables entre pays et de procéder à divers croisements avec les données sociodémographiques. Une étude de l'adéquation des compétences (ou des qualifications) aux emplois occupés permet de mesurer les taux de surqualification et de sous-qualification, deux situations contre-productives à terme.

Dans tous les pays, avec des degrés de corrélation variables, on observe un lien entre la maîtrise des compétences et les déclarations relatives à la santé et à la vie sociale. Les adultes ayant de faibles compétences en littératie indiquent plus souvent un état de santé moyen ou faible, font moins confiance à autrui, s'engagent peu dans le bénévolat et se sentent peu d'influence sur la vie politique du pays.

19 Face aux résultats de PIIAC, l'OCDE prodigue aux décideurs politiques des conseils pour élever les niveaux de compétence, en visant des gains au plan individuel - un faible niveau empêche d'accéder aux emplois rémunérateurs et gratifiants, aux services de base et aux offres de formation - comme au plan national car une trop forte proportion d'adultes peu compétents entrave les possibilités de faire évoluer organisations et techniques.

20 À l'évidence, une forte proportion de personnes compétentes s'observe dans les pays au plus fort revenu par habitant. Faut-il entendre que l'amélioration des compétences 
induirait mécaniquement une élévation du niveau de vie ? On pourrait en débattre car, inversement, c'est l'augmentation de la richesse nationale qui permet d'élever le niveau de formation.

21 Tout travailleur compétent sera-t-il employé à bon niveau? À terme peut-être, mais la fuite des cerveaux montre bien que le facteur temps a son importance. Si l'on doit saluer cette étude de l'OCDE, qui offre un état des lieux nouveau et suggère des décisions à prendre, on doit toutefois espérer que politiques, économistes, enseignants et sociologues s'emparent des données et fournissent des analyses et des recommandations nuancées et adaptées à chaque situation nationale.

\section{INDEX}

Index géographique : Pays de l'OCDE

Mots-clés : adulte, compétence, évaluation, formation continue, formation initiale, formation professionnelle, nouvelles technologies, littératie, numératie, PIAAC : programme pour l'évaluation internationale des compétences des adultes

\section{AUTEUR}

\section{ANNE-MARIE BARDI}

Anne-Marie Bardi est inspectrice générale honoraire de l'éducation nationale (France). Ancienne élève de l'École normale supérieure de Fontenay-aux-Roses, agrégée de mathématiques, elle a été professeur puis inspectrice d'académie-inspectrice pédagogique régionale de mathématiques. Dès les années 1970, elle s'est impliquée dans le développement de l'usage des technologies de l'information et de la communication dans l'enseignement en France et à l'étranger. 